\title{
Comparación de los espaciadores articulados vs. fijos en la revisión de la infección periprotésica de rodilla
}

\author{
Tomás I. Nicolino, Juan Astoul Bonorino, Agustín Molina, Julián Costantini, Matías Costa Paz, Lisandro Carbó \\ Sector de Artroscopia y Prótesis de Rodilla, Instituto de Ortopedia y Traumatología "Prof. Dr. Carlos E. Ottolenghi", \\ Hospital Italiano de Buenos Aires, Ciudad Autónoma de Buenos Aires, Argentina
}

\section{RESUMEN}

Introducción: Los espaciadores de cemento con antibiótico pueden ser fijos o articulados y se logra un resultado similar con ambos para erradicar una infección. Nuestro objetivo fue comparar el rango de movilidad articular y los resultados funcionales después del reimplante. Materiales y Métodos: Estudio de cohorte retrospectiva de pacientes sometidos a una revisión de la prótesis de rodilla por infección, en dos tiempos quirúrgicos. Se analizó la funcionalidad según el Knee Society Score (KSS) al año de la cirugía y se registró el rango de movilidad a los 45 días. Se registraron el grado de defecto óseo, dolor, satisfacción, las complicaciones y la recidiva de la infección. Resultados: Se incluyeron 103 pacientes (40 con espaciador articulado, 63 con espaciador fijo). El grupo con espaciador articulado tuvo una mediana 2,5 mayor en la movilidad final (102,5; RIC 95-110 vs. 100; RIC 90-105, p 0,01). Según el KSS funcional y el KSS de rodilla, no hubo diferencias entre ambos grupos. No hubo diferencias en el grado de satisfacción, dolor y el tiempo hasta el reimplante. Las complicaciones fueron similares en ambos grupos, con una tasa de reinfección sin diferencias estadísticamente significativas. Conclusión: Los espaciadores articulados proporcionaron un beneficio en el rango de movilidad después del reimplante de la prótesis.

Palabras clave: Artroplastia de rodilla; espaciadores; infección periprotésica; rango de movilidad articular.

Nivel de Evidencia: III

\section{Comparison Between Articulating vs. Fixed Spacers in Revision for Periprosthetic Knee Infection}

\begin{abstract}
Introduction: Cement spacers with antibiotics can be fixed or articulating, with similar results in eradicating infection. Our objective was to compare joint range of motion (ROM) and functional outcomes after reimplantation. Materials and Methods: A retrospective cohort study of patients who had undergone a knee prosthetic revision due to infection in two surgical stages. Functionality was analyzed according to the Knee Society Score (KSS) one year after surgery and ROM was recorded 45 days after surgery. Bone defect, pain, satisfaction, complications, and recurrence of infection were recorded. Results: A total of 103 patients were included. 40 with articulating spacers and 63 with fixed spacers. The articulating spacer group presents a median of 2.5 degrees greater in final mobility (102.5 IQR 95-110 vs 100 IQR 90-105, p 0.01). The KSS functional scale and KSS of the knee did not show differences between two groups. There were no differences concerning satisfaction, pain, and time until reimplantation. Complications were similar in both groups, with a reinfection rate without statistically significant differences. Conclusion: Articulating spacers have shown a benefit in ROM after prosthetic reimplantation.
\end{abstract}

Keywords: Knee arthroplasty; spacers; periprosthetic infection; joint range of motion.

Level of Evidence: III

Recibido el 10-6-2021. Aceptado luego de la evaluación el 8-7-2021 • Dr. TOMÁS I. NICOLINO • tomas.nicolino@ hospitalitaliano.org.ar

Cómo citar este artículo: Nicolino TI, Astoul Bonorino J, Molina A, Costantini J, Costa Paz M, Carbó L. Comparación de los espaciadores articulados vs. fijos en la revisión de la infección periprotésica de rodilla. Rev Asoc Argent Ortop Traumatol 2021;86(4):493-500. https://doi.org/10.15417/issn. 1852-7434.2021.86.4.1391 


\section{INTRODUCCIÓN}

La infección periprotésica luego de un reemplazo total de rodilla continúa siendo una de las complicaciones más temidas y tiene consecuencias que pueden llegar a ser devastadoras. La incidencia varía entre el $0,7 \%$ y el $2 \%$ a pesar de la mejoras de la técnica quirúrgica.

En la actualidad, la cirugía en dos tiempos para tratar la infección periprotésica articular colocando un espaciador de cemento cargado con antibiótico antes del reimplante de la prótesis es el tratamiento más utilizado y la tasa de erradicación de la infección es $>80-90 \%$. $^{2}$ Los espaciadores de cemento con antibiótico se pueden clasificar en dos tipos: fijos, que no permiten la movilidad articular de la rodilla y articulados, que mantienen cierto rango de movilidad durante su uso (Figura 1).

La confección de los espaciadores ha ido evolucionando y permanentemente se analizan las ventajas y desventajas de estos dos tipos de espaciadores. Las tasas de erradicación de la infección son similares independientemente del tipo de espaciador. Hoy se recomienda más el uso de espaciadores articulados sobre la base de una teórica mejoría de la movilidad articular. ${ }^{3-5}$

El objetivo principal de este estudio fue comparar el rango de movilidad articular y los resultados funcionales mediante el Knee Society Score (KSS) entre un grupo de pacientes tratados con un espaciador fijo y otro con un espaciador articulado.
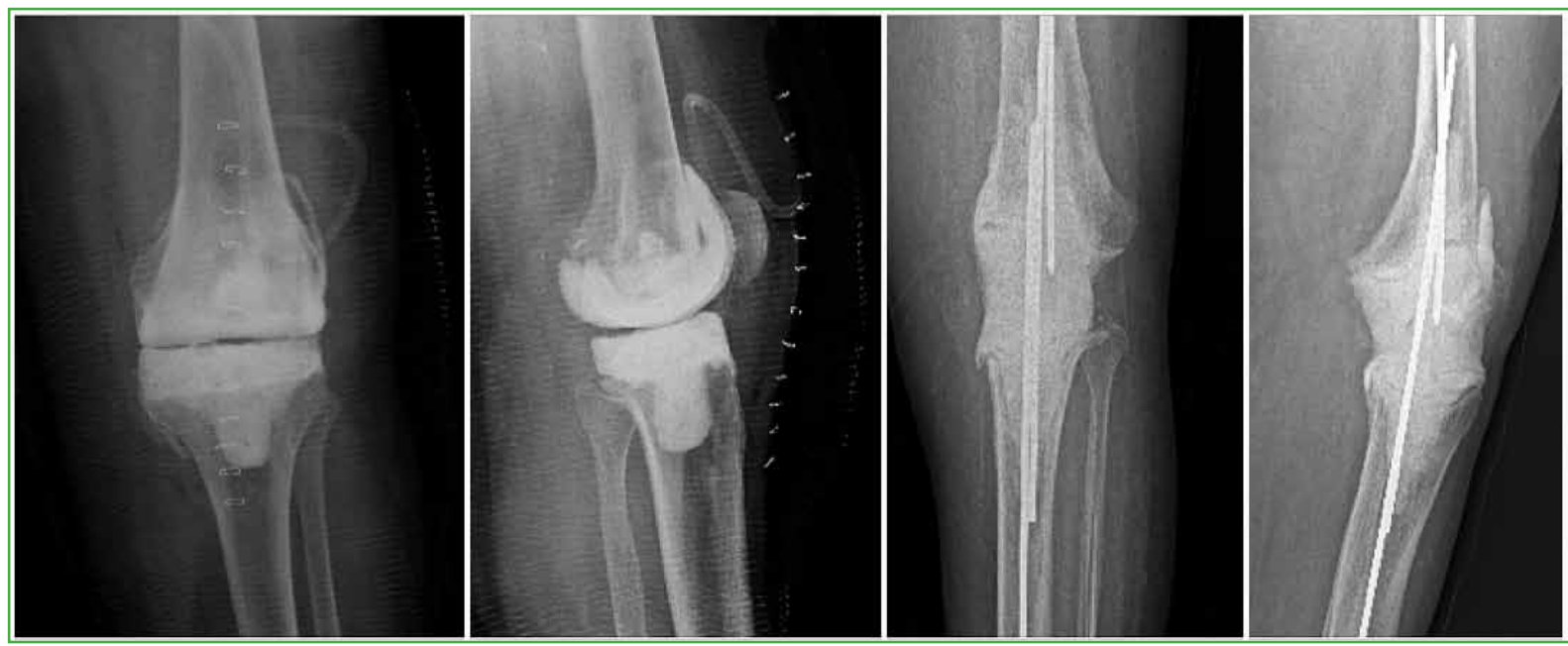

Figura 1. Espaciador articulado y espaciador fijo utilizados en esta serie.

\section{MATERIALES Y MÉTODOS}

\section{Diseño y recolección de datos}

Estudio de cohorte retrospectiva de pacientes que se sometieron a una revisión de la prótesis de rodilla por infección, en dos tiempos quirúrgicos, en un centro de referencia de alta complejidad.

Se analizaron los datos obtenidos de la historia clínica electrónica del período entre el 1 de enero de 2012 y el 1 de enero de 2019. La fecha de ingreso en el estudio fue la fecha de retiro de la prótesis infectada y se realizó el seguimiento hasta la última fecha de control alejado.

Se incluyó a todos los pacientes con infección periprotésica de rodilla tratados mediante la extracción de la prótesis y la colocación de un espaciador de cemento con antibiótico. Se excluyó a los pacientes que habían sido sometidos, como procedimiento final, a una artrodesis de rodilla. Se los clasificó en dos grupos: con espaciador fijo y con espaciador articulado.

\section{Variables analizadas}

Se evaluaron las características clínicas y demográficas de la serie. Para la evaluación del objetivo primario se analizó la funcionalidad tras el reimplante mediante el $\mathrm{KSS}^{6}$ al año de la operación y se registró con un goniómetro el rango de movilidad articular a los 45 días de la cirugía. 
Se registró la satisfacción del paciente durante el uso del espaciador utilizando una escala porcentual de satisfacción lineal ${ }^{7}$ aplicada un día antes de la segunda cirugía. Se registró el dolor durante el uso del espaciador mediante la escala analógica visual. ${ }^{8}$ Se evaluó la pérdida ósea utilizando la clasificación del Anderson Orthopaedic Research Institute (AORI), ${ }^{9}$ para la cual se registró el estado óseo luego del primer tiempo quirúrgico y en el momento del reimplante, en el segundo tiempo. Se evaluó el tiempo de uso del espaciador registrado como semanas que transcurrieron desde el primer tiempo quirúrgico hasta el segundo. Se registraron las complicaciones relacionadas con el uso del espaciador (rotura, luxación, rotura y luxación, reinfección/recambio del espaciador, trombosis venosa profunda). Como la muestra era fija, se incluyó a todos los pacientes que cumplieron con los criterios de selección durante el período de estudio.

\section{Análisis estadístico}

Las variables categóricas se describieron con número absoluto y porcentaje; y las variables numéricas, con mediana y percentil $25 \%-75 \%$. Las características entre los pacientes sometidos a uno u otro tratamiento se compararon con las pruebas de ji al cuadrado, Fisher o Mann-Witney, según correspondiera. Para explorar los factores asociados al rango de movilidad a los 45 días del reimplante, se realizó una regresión lineal múltiple, considerando el tipo de espaciador utilizado como variable de exposición, y la edad, el tiempo transcurrido hasta el reimplante, el grado de defecto AORI basal, y el grado de satisfacción y dolor al año de la cirugía como potenciales factores de confusión.

Para analizar el grado de defecto óseo inicial posresección y su progresión en el momento del reimplante de la prótesis, se utilizó la prueba de McNemar.

Todas las pruebas son a dos colas, y se consideró significativo un valor p $<0,05$. Se utilizó el programa Stata v14.

\section{Consideraciones éticas}

Este estudio se llevó a cabo respetando las consideraciones relativas al cuidado de los participantes en investigación clínica de acuerdo con las guías nacionales e internacionales incluidas en la Declaración de Helsinki.

\section{RESULTADOS}

Se incluyó a 103 pacientes. Cuarenta recibieron un espaciador articulado y 63, un espaciador fijo. La mediana de edad fue de 65 años (rango intercuartílico [RIC] 58-73), el grupo con espaciador articulado tenía una población ligeramente más joven (60.5; RIC 53-70 vs. 68; RIC 60-73; p 0,01). Las características clínicas y demográficas se detallan en la Tabla 1. Como se muestra en esta Tabla, no hubo diferencias en el tiempo transcurrido desde la colocación del espaciador hasta el reimplante, en ambos grupos.

Tabla 1. Variables demográficas de la población de estudio

\begin{tabular}{|c|c|c|c|c|}
\hline Variable & $\begin{array}{c}\text { Todos } \\
\mathrm{n}=103\end{array}$ & $\begin{array}{l}\text { Articulados } \\
\qquad n=40\end{array}$ & $\begin{array}{c}\text { Fijos } \\
n=63\end{array}$ & $\mathbf{p}$ \\
\hline Edad, mediana (RIC) & $65(58-73)$ & $60.5(53-70)$ & $68(60-73)$ & 0,01 \\
\hline Índice de masa corporal, mediana (RIC) & $28(26-31)$ & $28,5(26-31)$ & $28(26-30)$ & 0,51 \\
\hline Satisfacción, mediana (RIC) & $70(65-80)$ & $75(70-85)$ & $70(60-80)$ & 0,06 \\
\hline Dolor, mediana, escala analógica visual (RIC) & $3(2-4)$ & $4(3-5)$ & $3(2-4)$ & 0,03 \\
\hline Rango de movilidad posoperatorio, mediana (RIC) & $100(90-110)$ & $102,5(95-110)$ & $100(90-105)$ & 0,01 \\
\hline Tiempo al reimplante - semanas, mediana (RIC) & $10(9-11)$ & $10(9-11)$ & $10(9-11)$ & 0,3 \\
\hline Lado derecho, cantidad (\%) & $52(50,5)$ & $21(52,5)$ & $31(49,2)$ & 0,74 \\
\hline $\begin{array}{l}\text { Diagnóstico, cantidad (\%) } \\
\text { Infección periprotésica } \\
\text { Artrofibrosis }\end{array}$ & $\begin{array}{c}103(100) \\
101(98,1) \\
2(1,9)\end{array}$ & $\begin{array}{c}40(100) \\
38(95) \\
2(5)\end{array}$ & $\begin{array}{c}63(100) \\
63(100) \\
0(0 \%)\end{array}$ & 0,07 \\
\hline
\end{tabular}

RIC = rango intercuartílico. 


\section{Evaluación del rango de movilidad}

$\mathrm{Al}$ evaluar el rango de movilidad articular a los 45 días del reimplante, se observaron mejores resultados en el grupo con espaciadores articulados. Los pacientes sometidos a esta técnica tuvieron una mediana de $2,5^{\circ}$ más en la movilidad final que los pacientes con espaciadores fijos (102,5; RIC 95-110 vs. 100; RIC 90-105, respectivamente; p 0,01).

El análisis multivariado de regresión lineal mostró que los pacientes con un espaciador fijo tuvieron, en promedio, 5,6 menos de movilidad que aquellos con espaciadores móviles, independientemente de la edad, el tiempo transcurrido hasta el reimplante, el grado de defecto AORI basal, y el grado de satisfacción y dolor al año de la cirugía (IC95\% de -10,03 a -1,33; p 0,011).

\section{Evaluación de los resultados funcionales}

Los resultados según el KSS funcional y el KSS de rodilla no difirieron entre ambos grupos. Para la evaluación del KSS funcional, el puntaje de los espaciadores articulados y el de los fijos fue de 78 (RIC 65-85) vs. 78 (RIC 70-82), respectivamente (p 0,82 ).

El KSS de rodilla fue de 85 (RIC 80-90) en ambos grupos (p 0,52) (Tabla 2).

Tabla 2. Resultados funcionales

\begin{tabular}{|l|l|l|l|l|}
\hline Variable & \multicolumn{1}{|c|}{$\begin{array}{c}\text { Todos } \\
\mathbf{n = 1 0 3}\end{array}$} & $\begin{array}{c}\text { Articulados } \\
\mathbf{n = 4 0}\end{array}$ & $\begin{array}{c}\text { Fijos } \\
\mathbf{n = 6 3}\end{array}$ \\
\hline KSS funcional, mediana (RIC) & $78(70-84)$ & $78(65-85)$ & $78(70-82)$ & 0,82 \\
\hline KSS rodilla, mediana (RIC) & $85(80-90)$ & $85(80-90)$ & $85(80-90)$ & 0,52 \\
\hline
\end{tabular}

KSS $=$ Knee Society Score, RIC $=$ rango intercuartílico.

Al evaluar el grado de satisfacción con los distintos espaciadores utilizados, el grupo de pacientes con un espaciador articulado refirió mayor satisfacción (75/100, RIC 70-85) que el grupo con espaciador fijo (70/100, RIC 60-80), la diferencia no fue estadísticamente significativa (p 0,06).

En la Tabla 3 y en la Figura 2, se muestra el grado de defecto óseo después de la resección y su progresión hasta el momento del reimplante.

Tabla 3. Grado de defecto óseo según AORI posresección y evolución del defecto antes del reimplante de la prótesis

\begin{tabular}{|l|c|c|c|}
\hline Variable & $\begin{array}{c}\text { Todos } \\
\mathbf{n}=\mathbf{1 0 3}\end{array}$ & $\begin{array}{c}\text { Articulados } \\
\mathbf{n}=\mathbf{4 0}\end{array}$ & $\begin{array}{c}\text { Fijos } \\
\mathbf{n}=\mathbf{6 3}\end{array}$ \\
\hline AORI posresección, cantidad (\%) & $103(100)$ & $40(100)$ & $63(100)$ \\
\hline 1 & $14(13,6)$ & $7(17,5)$ & $7(11,1)$ \\
\hline 2 & $31(30,1)$ & $18(45)$ & $13(20,6)$ \\
\hline 3 & $58(56,3)$ & $15(37,5)$ & $43(68,2)$ \\
\hline AORI antes del reimplante, cantidad (\%) & $103(100)$ & $40(100)$ & $63(100)$ \\
\hline 1 & $6(5,8)$ & $2(5)$ & $4(6,3)$ \\
\hline 3 & $28(27,2)$ & $16(40)$ & $12(19,1)$ \\
\hline
\end{tabular}

AORI = Anderson Orthopaedic Research Institute, RIC = rango intercuartílico. 


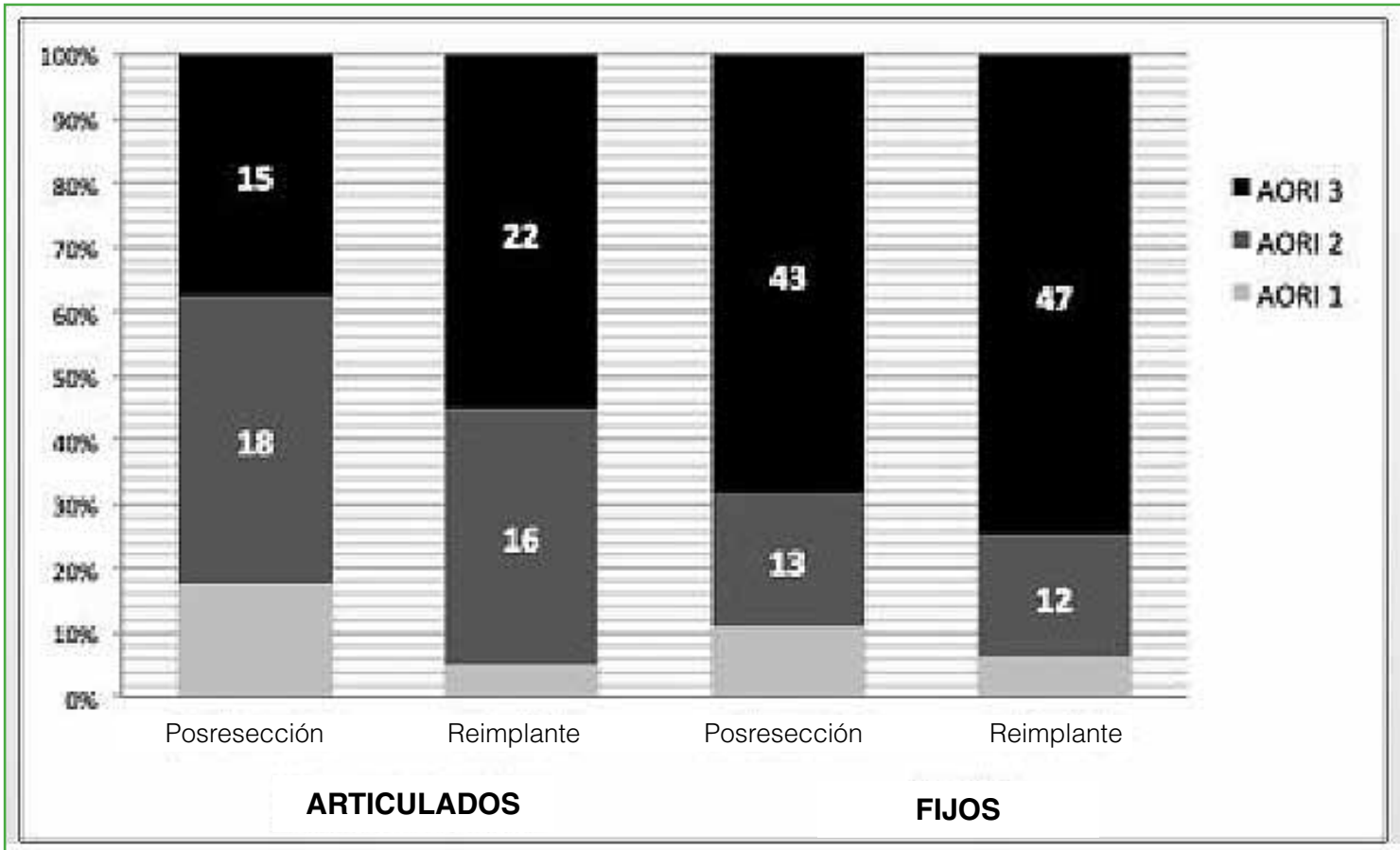

Figura 2. Grado de defecto óseo según el tipo de espaciador. Evaluación posresección y evolución del defecto antes del reimplante de la prótesis.

Por último, no se observaron diferencias en la frecuencia de complicaciones entre ambos grupos en cuanto a rotura o luxación del espaciador, reinfección o trombosis venosa profunda (Tabla 4, Figura 3).

Tabla 4. Complicaciones registradas en ambos grupos durante el uso del espaciador

\begin{tabular}{|l|c|c|c|}
\hline Variable & $\begin{array}{c}\text { Todos } \\
\mathbf{n}=\mathbf{1 0 3}\end{array}$ & $\begin{array}{c}\text { Articuladijos } \\
\mathbf{n}=\mathbf{4 0}\end{array}$ & $\begin{array}{c}\text { Fijos } \\
\mathbf{n}=\mathbf{6 3}\end{array}$ \\
\hline Complicaciones del espaciador (\%) & $15(14,6)$ & $7(17,5)$ & $8(12,7)$ \\
\hline Rotura & $5(33,3)$ & $3(42,8)$ & $2(25)$ \\
\hline Luxación & $2(13,3)$ & $1(14,3)$ & $1(12,5)$ \\
\hline Rotura más luxación & $1(6,8)$ & $1(14,3)$ & $1(12,5)$ \\
\hline Infección & $2(13,3)$ & $2(28,6)$ & $3(37,5)$ \\
\hline Trombosis venosa profunda & $5(33,3)$ & & \\
\hline
\end{tabular}




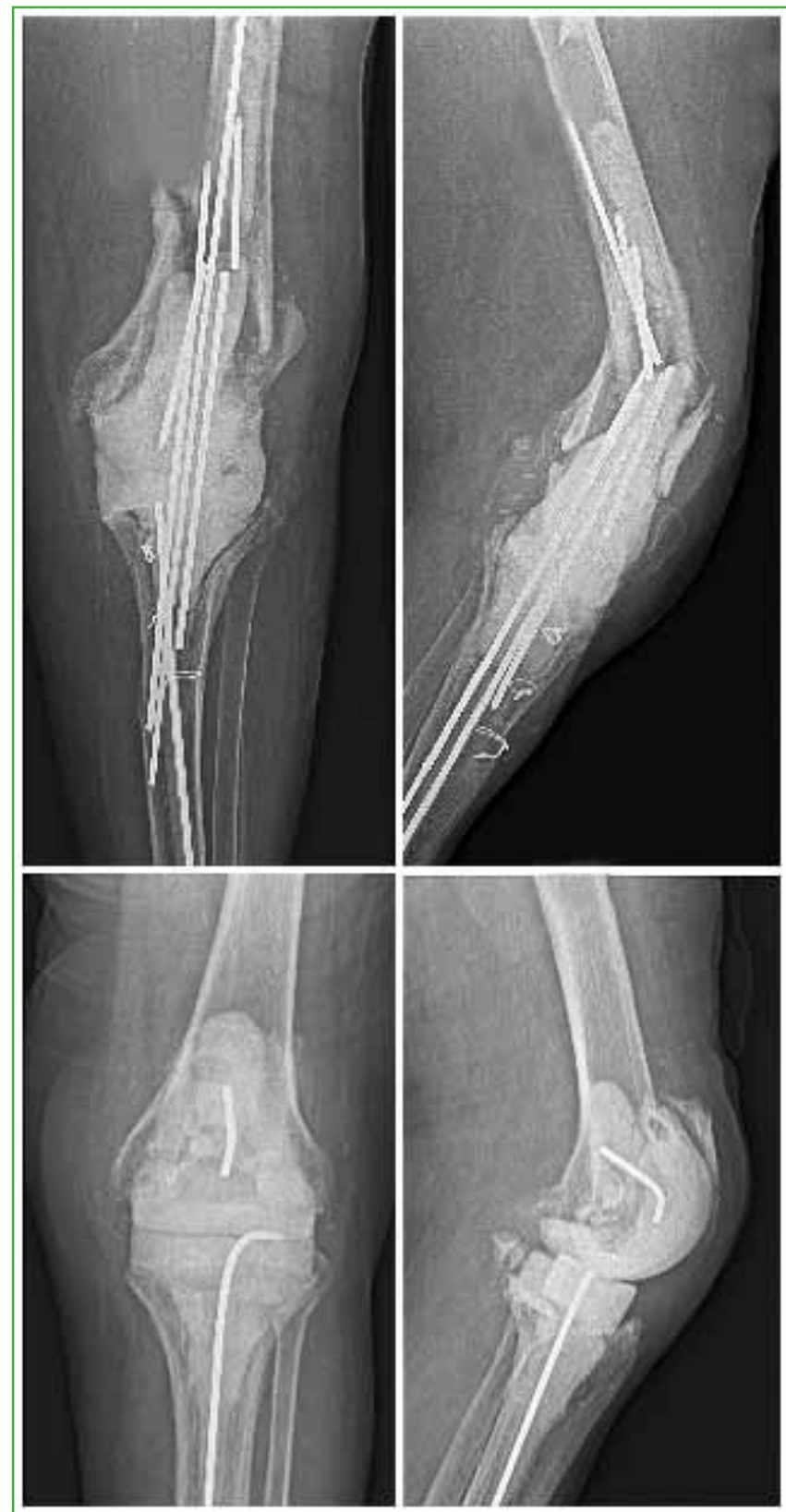

Figura 3. Complicaciones registradas en nuestra serie. Luxación del espaciador móvil y rotura del espaciador fijo.

\section{DISCUSIÓN}

Dado al aumento de la población mundial, de la expectativa de vida y, por lo tanto, de la cantidad de reemplazos articulares que se realizan, ${ }^{10}$ la infección periprotésica asociada al reemplazo total de rodilla es un gran desafío para el que debemos estar preparados.

Cuando la elección terapéutica es una revisión en dos tiempos, la cirugía de extracción de la prótesis y la colocación de un espaciador adquieren gran relevancia. El espaciador utilizado debe cumplir con las condiciones para facilitar el segundo tiempo quirúrgico y sobre todo proporcionar un tratamiento adecuado para erradicar la infección y lograr los resultados funcionales esperados tras el reimplante. 
El rol que cumple el tipo de espaciador en el resultado tras el reimplante, tanto en el rango de movilidad como en los resultados funcionales, ha sido tema de debate. La evidencia actual ha marcado una recomendación hacia el uso de espaciadores articulados, porque estos favorecen la movilidad posoperatoria. ${ }^{11}$ Pivec y cols. informaron que el rango de movilidad promedio final en una población de 962 pacientes con espaciadores articulados fue superior que en otros 707 pacientes con espaciadores fijos. Nuestros resultados confirman lo expuesto en este estudio; además, coinciden en que no se demostró una diferencia en cuanto a la evaluación funcional según el KSS. ${ }^{4}$ Esta recomendación de utilizar espaciadores articulados, cuando las condiciones operatorias lo permitan, fue incluso establecida durante el consenso de Filadelfia, en 2013. ${ }^{12}$

La correcta selección del espaciador debe considerar, entre otros factores, las condiciones intraoperatorias durante el primer tiempo quirúrgico, específicamente el grado de defecto óseo. Por ello, en nuestra población de pacientes con defectos óseos severos (AORI 3), la indicación de un espaciador fijo prevaleció sobre la de uno articulado. Sin embargo, la progresión de los defectos óseos AORI 1 y 2 hasta el momento del reimplante fue similar en ambos grupos.

Por último, las revisiones sistemáticas publicadas, como la de Voleti y cols., donde se analizan 1526 pacientes en estudios con nivel de evidencia III y IV, revelaron que no existen diferencias en las tasas de reinfección según el tipo de espaciador empleado ( $12 \%$ espaciadores fijos vs. $8 \%$ articulados, p 0,01$){ }^{13,14}$ Nuestros resultados coinciden con esta afirmación; a su vez, no hubo diferencias significativas en las complicaciones relacionadas con el tipo de espaciador empleado entre ambos grupos.

Una debilidad de este estudio es su carácter retrospectivo; las indicaciones de un espaciador u otro pueden depender del médico tratante, y del tipo de defecto y el paciente por tratar. Como fortaleza mencionamos que se trata de una serie con más de 100 pacientes tratados por un mismo equipo, sin pérdidas en el seguimiento.

\section{CONCLUSIONES}

Los espaciadores articulados para el tratamiento de las infecciones de la prótesis de rodilla han proporcionado un beneficio en el rango de movilidad articular después del reimplante de la prótesis. Los resultados funcionales, según el KSS, el grado de satisfacción y dolor, y la recidiva de la infección al año de la cirugía no difirieron entre ambos grupos.

Conflicto de intereses: Los autores no declaran conflictos de intereses.

ORCID de J. Astoul Bonorino: https://orcid.org/0000-0001-6798-8242

\section{BIBLIOGRAFÍA}

1. Lachiewicz PF, Wellman SS, Peterson JR. Antibiotic cement spacers for infected total knee arthroplasties. $J A m$ Acad Orthop Surg 2020;28:180-8. https://doi.org/10.5435/JAAOS-D-19-00332

2. Warth LC, Hadley CJ, Grossman EL. Two-stage treatment for total knee arthroplasty infection utilizing an articulating prefabricated antibiotic spacer. J Arthroplasty 2020;35:S57-S62. https://doi.org/10.1016/j.arth.2019.10.049

3. Guild GN 3rd, Wu B, Scuderi GR. Articulating vs. static antibiotic impregnated spacers in revision total knee arthroplasty for sepsis. A systematic review. J Arthroplasty 2014;29: 558-63. https://doi.org/10.1016/j.arth.2013.08.013

4. Pivec R, Naziri Q, Issa K, Banerjee S, Mont MA. Systematic review comparing static and articulating spacers used for revision of infected total knee arthroplasty. J Arthroplasty 2014;29:553-7.e1.

https://doi.org/10.1016/j.arth.2013.07.041 
5. Mazzucchelli L, Rosso F, Marmotti A, Bonasia DE, Bruzzone M, Rossi R. The use of spacers (static and mobile) in infection knee arthroplasty. Curr Rev Musculoskelet Med 2015;8:373-82. https://doi.org/10.1007/s12178-015-9293-8

6. Liow RY, Walker K, Wajid MA, Bedi G, Lennox CM. The reliability of the American Knee Society Score. Acta Orthop Scand 2000;71:603-8. https://doi.org/10.1080/000164700317362244

7. Kahlenberg CA, Nwachukwu BU, McLawhorn AS, Cross MB, Cornell CN, Padgett DE. Patient satisfaction after total knee replacement: a systematic review. HSS J 2018;14:192-201. https://doi.org/10.1007/s11420-018-9614-8

8. Langley GB, Sheppeard H. The visual analogue scale: its use in pain measurement. Rheumatol Int 1985;5:145-8. https://doi.org/10.1007/BF00541514

9. Engh GA, Parks NL. The management of bone defects in revision total knee arthroplasty. Instr Course Lect 1997;46:227-36. Disponible en: https://www.ncbi.nlm.nih.gov/pubmed/9143967

10. Arden N, Nevitt MC. Osteoarthritis: epidemiology. Best Pract Res Clin Rheumatol 2006;20:3-25. https://doi.org/10.1016/j.berh.2005.09.007

11. Wyles CC, Abdel MP. Point/Counterpoint: Nonarticulating vs articulating spacers for resection arthroplasty of the knee or hip. J Arthroplasty 2020;35:S40-S44. https://doi.org/10.1016/j.arth.2019.10.055

12. Parvizi J, Gehrke T, Chen AF. Proceedings of the International Consensus on Periprosthetic Joint Infection. Bone Joint J 2013;95B:1450-2. https//doi.org/10.1302/0301-620X.95B11.33135

13. Voleti PB, Baldwin KD, Lee G-C. Use of static or articulating spacers for infection following total knee arthroplasty: a systematic literature review. J Bone Joint Surg Am 2013;95:1594-9. https://doi.org/10.2106/JBJS.L.01461

14. Bowman EC, Malkani AL. Point/Counterpoint: Static vs articulating spacers-static spacers for resection arthroplasty of the knee. J Arthroplasty 2020;35:S35-S39. https://doi.org/10.1016/j.arth.2019.10.033 